Case Report

\title{
Quetiapine-Induced Syndrome of Inappropriate Secretion of Antidiuretic Hormone
}

\author{
Theocharis Koufakis \\ Department of Internal Medicine, General Hospital of Larissa, 1 Tsakalof Street, 41221 Larissa, Greece
}

Correspondence should be addressed to Theocharis Koufakis; thkoyfak@hotmail.com

Received 23 December 2015; Revised 7 February 2016; Accepted 15 February 2016

Academic Editor: Toshiya Inada

Copyright (C) 2016 Theocharis Koufakis. This is an open access article distributed under the Creative Commons Attribution License, which permits unrestricted use, distribution, and reproduction in any medium, provided the original work is properly cited.

The syndrome of inappropriate secretion of antidiuretic hormone (SIADH) can be induced by various conditions, including malignant neoplasms, infections, central nervous system disorders, and numerous drugs. We here report a case of a 65-year-old female patient, treated with quetiapine for schizophrenia, who presented with generalized tonic-clonic seizures and was finally diagnosed with quetiapine-induced SIADH. Quetiapine-associated hyponatremia is extremely uncommon and only a few, relevant reports can be found in the literature. This case underlines the fact that patients on antipsychotic medication and more specifically on quetiapine should be closely monitored and routinely tested for electrolyte disorders.

\section{Introduction}

The syndrome of inappropriate secretion of antidiuretic hormone (SIADH) is defined by hypotonic hyponatremia, inappropriately elevated urine osmolality relative to plasma osmolality, an elevated urine sodium level, expanded extracellular volume, and normal renal, adrenal, and thyroid function [1]. SIADH can be induced by various conditions, including malignant neoplasms, infections (especially pulmonary ones), central nervous system disorders, and numerous drugs [2].

Quetiapine is an antipsychotic agent, widely used for the treatment of schizophrenia, bipolar disorder, and major depressive disorder. Frequently reported side effects of the drug include dizziness, dry mouth, nausea, constipation, lethargy, and increased appetite. Prolonged QT interval [3] and hematological effects [4] have been also described as unusual adverse reactions of quetiapine use. Still, quetiapineassociated hyponatremia is generally uncommon and only a few, relevant reports can be found in the literature.

\section{Case Report}

A 65-year-old female patient presented to the Emergency Department with generalized tonic-clonic seizures. She had been diagnosed with schizophrenia at the age of 40 and she was on quetiapine (300 $\mathrm{mg}$ daily, orally, divided into 3 doses), since 3 months. She denied consumption of any other drug, dry mouth symptom, and present or past history of excessive water drinking. The latter was also documented by interviewing patient's close relatives. Other than schizophrenia, her medical history was unremarkable for chronic diseases.

The patient was normotensive (blood pressure $130 / 85 \mathrm{mmHg}$ ). Physical examination did not reveal any abnormal findings. Peripheral oedema was absent. Her main laboratory findings on admission were as follows: serum sodium concentration $108 \mathrm{mmol} / \mathrm{L}(135-145 \mathrm{mmol} / \mathrm{L})$, serum osmolarity $243 \mathrm{mOsm} / \mathrm{L}(275-295 \mathrm{mOsm} / \mathrm{L})$, urine sodium concentration $68 \mathrm{mmol} / \mathrm{L}(<20 \mathrm{mmol} / \mathrm{L})$, and urine osmolality $264 \mathrm{mOsm} / \mathrm{kg}$. Renal, liver, and thyroid function tests as well as cortisol levels proved to be within the normal limits.

In view of these findings, the diagnosis of SIADH was established, according to the criteria described by Bartter and Schwartz [1] (Table 1). During her hospitalization, a complete diagnostic workup was performed, including thorough laboratory testing, brain, chest, and abdomen CT scans and gastrointestinal endoscopy. The above diagnostic procedures excluded other factors as the potential causes of the syndrome, such as malignancies, infections, and stroke.

Seizures were attributed to severe hyponatremia and were treated with diazepam. Initial management of the patient at the Emergency Department included intravenous infusion 
TABLE 1: The criteria required for the diagnosis of SIADH and the main clinical and laboratory findings of the presented case, which led to the diagnosis of the syndrome.

\begin{tabular}{ll}
\hline Criteria needed for SIADH diagnosis & Patient's laboratory and clinical findings \\
\hline $\begin{array}{l}\text { (1) Decreased plasma osmolality } \\
(<275 \mathrm{mOsm} / \mathrm{kg})\end{array}$ & Plasma osmolarity $243 \mathrm{mOsm} / \mathrm{L}(275-295 \mathrm{mOsm} / \mathrm{L})$ \\
$\begin{array}{l}\text { (2) Inappropriately concentrated urine } \\
(>100 \mathrm{mOsm} / \mathrm{kg})\end{array}$ & Urine osmolality $264 \mathrm{mOsm} / \mathrm{kg}$ \\
$(3)$ Being euvolemic & $\begin{array}{l}\text { The patient was normotensive (blood pressure } 130 / 85 \mathrm{mmHg}) . \text { Physical } \\
\text { examination did not reveal any abnormal findings. Peripheral oedema was absent }\end{array}$ \\
(4) Elevated urine $\mathrm{Na}(>20 \mathrm{mEq} / \mathrm{L})$ & $\begin{array}{l}\text { Urine Na concentration } 68 \mathrm{mEq} / \mathrm{L} \\
\text { (5) Euthyroid, eucortisolemic, and no diuretic }\end{array}$ \\
use. & $\begin{array}{l}\text { Renal, liver, and thyroid function tests and cortisol levels were within the normal } \\
\text { limits. Comedication was not present. Patient's medical history was unremarkable } \\
\text { for chronic diseases }\end{array}$ \\
\hline
\end{tabular}

of $150 \mathrm{~mL}$ of $3 \%$ hypertonic saline $(\mathrm{NaCl})$ solution over 20 minutes. After repeating the same procedure over the next 20 minutes, serum sodium concentration was measured again and found to be $113 \mathrm{mmol} / \mathrm{L}$. Subsequently, fluids limitation (500 $\mathrm{mL}$ 0.9\% saline daily) and quetiapine withdrawal resulted in the restoration of serum sodium concentration and plasma osmolarity to the normal levels, within the next 72 hours $(135 \mathrm{mmol} / \mathrm{L}$ and $285 \mathrm{mOsm} / \mathrm{L}$, resp.). The patient was discharged on olanzapine and, in her follow-up visits, she remained in good physical condition and her blood tests were all within the normal range.

\section{Discussion}

A wide variety of drugs has been previously accused of inducing SIADH, especially carbamazepine, selective serotonin reuptake inhibitors (SSRIs), and phenothiazines [5]. Both the newer atypical antipsychotics and the older drugs have been associated with the development of the syndrome [6]. The exact pathophysiological background of drug-induced SIADH is still unclear. However, stimulation of $\mathrm{ADH}$ release and increase of $\mathrm{ADH}$ renal action are believed to be the most probable mechanisms [2].

In most cases of SIADH associated with drugs, patients have mild, asymptomatic hyponatremia [2], which is usually detected once blood tests are ordered for an apparently irrelevant reason. However, several deaths related to hyponatremia induced by ecstasy (methylenedioxymethamphetamine or MDMA) have been reported. Moreover, cyclophosphamideand carbamazepine-induced SIADH have been linked with high mortality [7]. In the reported case, the patient's life is endangered due to seizures triggered by severe hyponatremia.

Management of drug-induced SIADH primarily demands the discontinuation of the suspected agent. In cases of severe hyponatremia, additional actions such as fluid restriction and furosemide administration should be taken [2]. Agents causing nephrogenic diabetes insipidus, such as demeclocycline and lithium carbonate, have been also widely used for the treatment of the syndrome.

In the described case, our initial approach was treating patient with prompt infusion of hypertonic 3\% saline solution, due to extremely low sodium serum levels and severe neurological symptomatology. After a $5 \mathrm{mmol} / \mathrm{L}$ increase of serum sodium concentration and stabilization of patient's neurological status, we continued with a diagnosis-specific, more conservative approach regarding the correction of sodium levels. Our decision was based, firstly, on our suspicion that SIADH and quetiapine were associated and, secondly, on our belief that patient's hyponatremia had been established more than 48 hours ago ("chronic" hyponatremia), given that the patient was on quetiapine for the past three months. It is generally accepted that symptomatic patients with severe hyponatremia should be treated aggressively to reduce cerebral edema and avoid herniation. However, rapid correction of serum sodium can result in central pontine myelinolysis and severe, irreversible neurological complications, such as spastic quadriparesis. Patients with chronic hyponatremia are at greater risk from rapid sodium correction, when compared with those with recently $(<48$ hours) established hyponatremia [8].

Another common cause of hyponatremia in schizophrenic patients is psychogenic polydipsia. Its distinction from SIADH should be based on history of excessive water consumption and opposite results of laboratory tests, such as urine osmolality and urine sodium levels [9].

Quetiapine-induced SIADH is extremely uncommon, as concluded from the few relevant reports found in the medical literature. Still, the exact prevalence of the above association is practically unknown. Moreover, the possibility of underdiagnosis and underreporting of this condition cannot be excluded. To our best knowledge, this is the third report in the literature, following the cases described by Atalay et al. [10] and van den Heuvel et al. [11]. Worth pointing out is the fact that, in both cases, serum sodium levels of the patients were significantly higher than the value measured in our case (128 and $120 \mathrm{mmol} / \mathrm{L}$, resp.). In conclusion, physicians should be aware of this rare, still severe adverse reaction of quetiapine. Patients on antipsychotic medication and more specifically on quetiapine should be closely monitored and routinely tested for electrolyte disorders.

\section{Conflict of Interests}

The author declares that there is no conflict of interests regarding the publication of this paper. 


\section{References}

[1] F. C. Bartter and W. B. Schwartz, "The syndrome of inappropriate secretion of antidiuretic hormone," The American Journal of Medicine, vol. 42, no. 5, pp. 790-806, 1967.

[2] A. M. Ramos-Levi, A. Duran Rodriguez-Hervada, M. MendezBailon, and J. Marco-Martinez, "Drug-induced hyponatremia: an updated review," Minerva Endocrinologica, vol. 39, no. 1, pp. 1-12, 2014.

[3] J. Nielsen, J. Matz, A. Mittoux et al., "Cardiac effects of sertindole and quetiapine: analysis of ECGs from a randomized double-blind study in patients with schizophrenia," European Neuropsychopharmacology, vol. 25, no. 3, pp. 303-311, 2015.

[4] K. Y. Fan, W. Y. Chen, and M. C. Huang, "Quetiapine-associated leucopenia and thrombocytopenia: a case report," BMC Psychiatry, vol. 15, article 110, 2015.

[5] S. Karahan, H. Karagöz, A. Erden, D. Avci, and K. Esmeray, "Codeine-induced syndrome of inappropriate antidiuretic hormone: case report," Balkan Medical Journal, vol. 31, no. 1, pp. 107-109, 2014.

[6] D. Meulendijks, C. K. Mannesse, P. A. F. Jansen, R. J. van Marum, and T. C. G. Egberts, "Antipsychotic-induced hyponatraemia: a systematic review of the published evidence," Drug Safety, vol. 33, no. 2, pp. 101-114, 2010.

[7] W. Fenske and B. Allolio, "The syndrome of inappropriate secretion of antidiuretic hormone: diagnostic and therapeutic advances," Hormone and Metabolic Research, vol. 42, no. 10, pp. 691-702, 2010.

[8] G. Spasovski, R. Vanholder, B. Allolio et al., "Clinical practice guideline on diagnosis and treatment of hyponatraemia," Nephrology Dialysis Transplantation, vol. 29, supplement 2, pp. i1-i39, 2014.

[9] B. Dundas, M. Harris, and M. Narasimhan, "Psychogenic polydipsia review: etiology, differential, and treatment," Current Psychiatry Reports, vol. 9, no. 3, pp. 236-241, 2007.

[10] A. Atalay, N. Turhan, and O. E. Aki, "A challenging case of syndrome of inappropriate secretion of antidiuretic hormone in an elderly patient secondary to quetiapine," Southern Medical Journal, vol. 100, no. 8, pp. 832-833, 2007.

[11] O. A. van den Heuvel, P. M. Bet, E. W. C. M. van Dam, and A. M. Eeckhout, "The syndrome of inappropriate antidiuretic hormone secretion (SIADH) during treatment with the antipsychotic agents haloperidol and quetiapine," Nederlands Tijdschrift voor Geneeskunde, vol. 150, no. 35, pp. 1944-1948, 2006. 


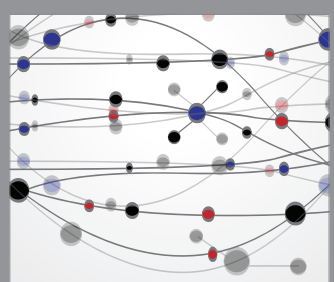

The Scientific World Journal
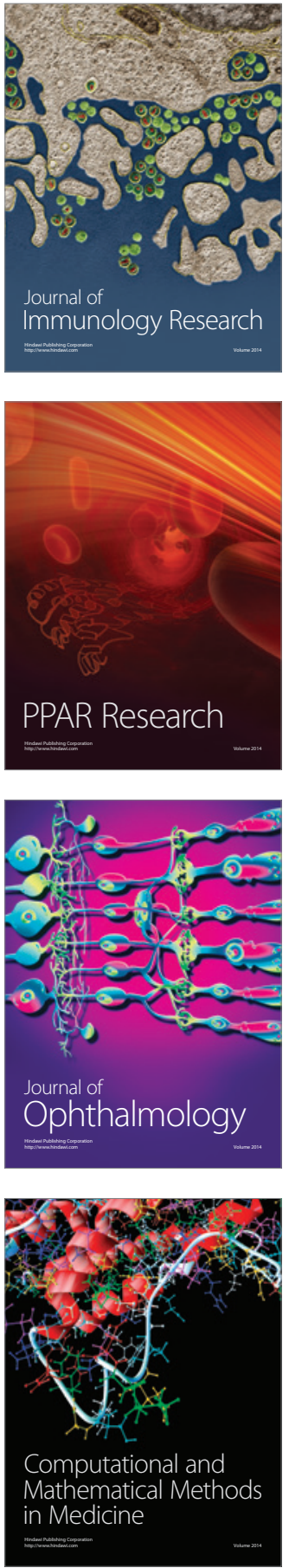

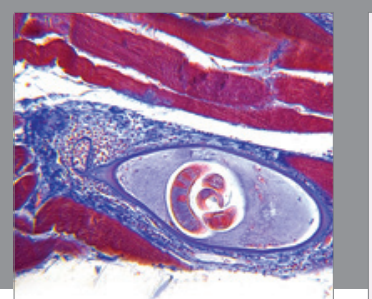

Gastroenterology Research and Practice

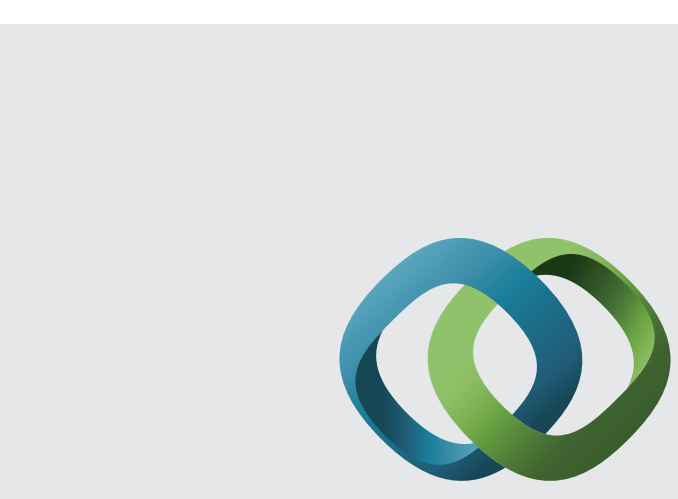

\section{Hindawi}

Submit your manuscripts at

http://www.hindawi.com
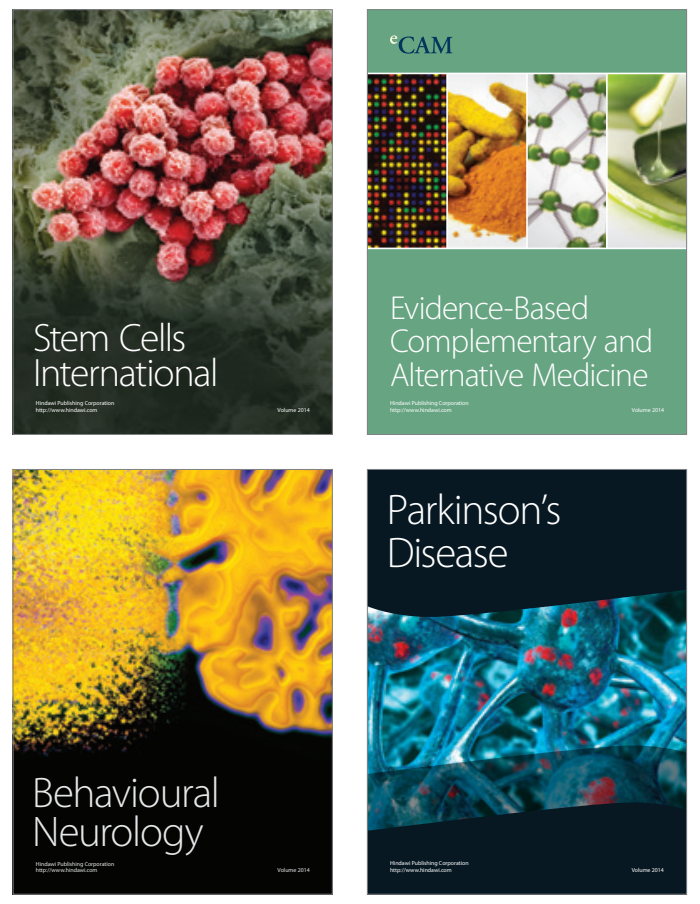
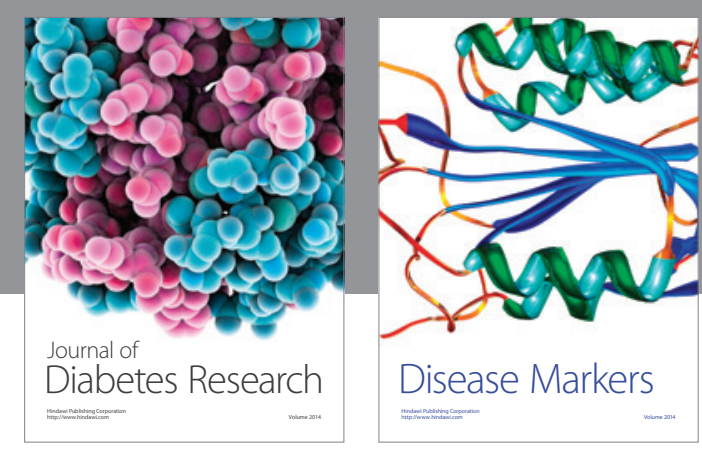

Disease Markers
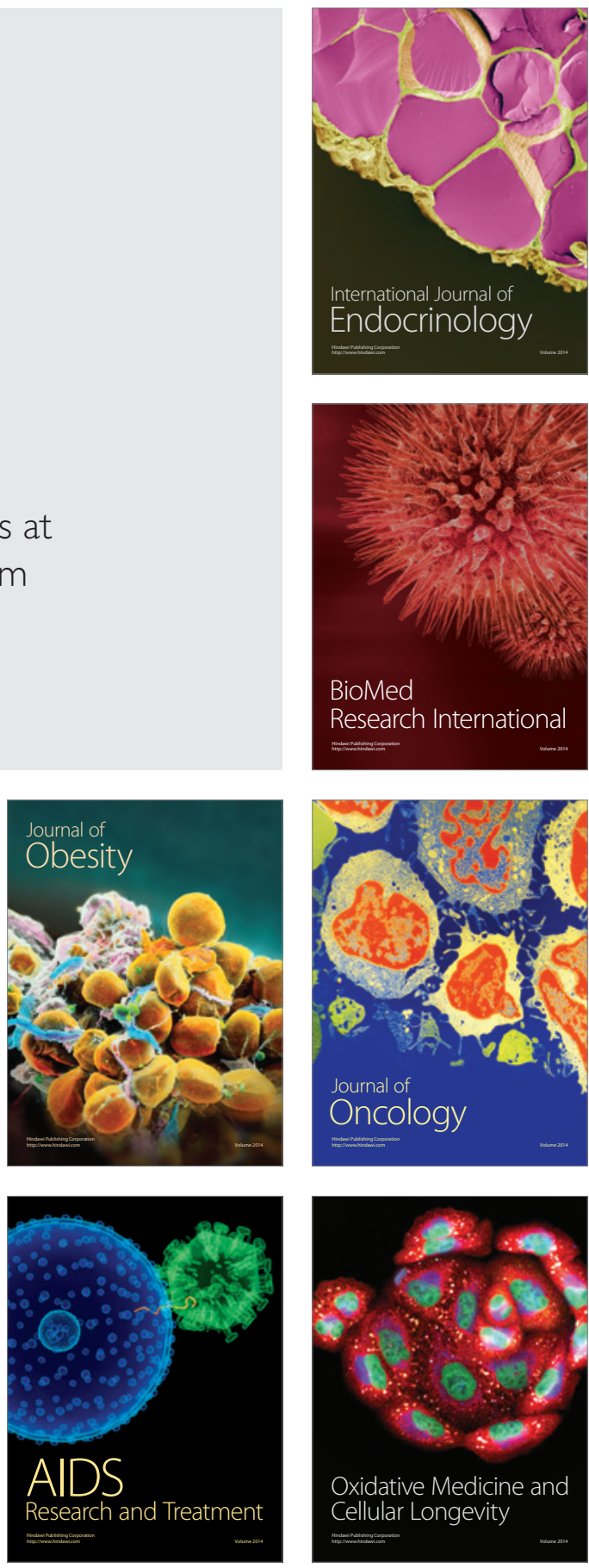